\title{
El posicionamiento de las marcas de automóviles en los estudiantes universitarios de la UEMC: diferencias de percepción en función de la experiencia en la conducción
}

\author{
Recibido: 10 de diciembre de 2010 \\ Aceptado: 27 de junio de 2011 \\ Publicado: 31 de octubre de 2011
}

\author{
Alejandro Tapia Frade \\ atapia@uemc.es \\ Universidad Europea Miguel de Cervantes (España)
}

Resumen: En este trabajo se realiza un análisis del posicionamiento de las marcas de automóviles sobre una muestra de estudiantes universitarios de la UEMC. El método estadístico utilizado fue el análisis de correspondencias simple en el marco de una encuesta sobre la muestra antes mencionada.

Los resultados ponen de manifiesto la estructuración del mercado de automóviles en todos los casos en varios grupos competitivos, en función fundamentalmente del precio. Además, se encontraron diferencias relevantes en el segmento de gama media en los dos períodos analizados, tanto en el caso de estudiantes con permiso de conducción como en el caso de estudiantes sin tal autorización administrativa.

Palabras clave: Automoción, imagen, posicionamiento, marcas, imagen, análisis de correspondencias.

Abstract: This paper provides an analysis of car brands positioning on a sample of UEMC university students. The statistical method used was simple correspondence analysis as part of a survey on the sample above mentioned.

The results reveal the structure of the automobile market in all cases where several competing groups depending on the price. We also found significant differences in the midrange segment in both periods analyzed, both in the case of students driving license as in the case of students without such authorization. 
Key words: Automotive, Image, Positioning, Brands, Image, Correspondence Analysis.

\section{Introducción}

La crisis económica de los últimos años ha afectado sensiblemente al consumo interno de este bien en España, que ha venido descendiendo notoriamente en los últimos años, si bien hay que hacer notar que en la Península la mayoría de los vehículos fabricados tienen como destino la exportación. No obstante, la situación de crisis ha sido en los últimos años general en el entorno de la Unión Europea, lo cual supone, en todo caso, un grave varapalo para el sector.

Además, en España es particularmente importante el sector de la automoción, ya que dicho sector representa el 3.5\% del PIB y es responsable del $8.7 \%$ del empleo existente en este país (Díaz de Alda, 2009), siendo uno de los mayores productores de vehículos, tanto en el ámbito de la Unión Europea como en el conjunto mundial. Por ello, no es de extrañar el elevado nivel de conocimiento hacia las distintas marcas por parte de los consumidores, explicado también por el alto número de publicaciones que sobre el sector existen. Así, la Oficina de Justificación de la difusión controla 27 cabeceras impresas del sector (OJD, 2009).

Así, el punto de partida de este trabajo parte de la conveniencia para las empresas del sector automovilístico de conocer el posicionamiento que poseen sus marcas en un segmento concreto de la demanda de automóviles (los estudiantes universitarios) para así poder llevar a cabo estrategias tanto comunicativas como operativas que se acerquen a dicho ideal y presentar una oferta atractiva a dicho segmento de consumo.

Los estudiantes universitarios suponen una interesante fuente de conocimiento ya que, en principio, hay que considerar que tienen un recorrido vital amplio, lo cual permite suponer que, al menos, una parte importante de ellos serán consumidores de automóviles en el medio plazo y durante un período de tiempo amplio.

En todo caso, el término "posicionamiento" debe, por tanto, entenderse en este trabajo desde el lado de la demanda, que según Trout y Ries (1972) se relaciona con el lugar, situación o emplazamiento ocupado por un objeto, una idea, una institución, un servicio, una ciudad o cualquier otra realidad susceptible de ser colocada respecto a otras con las que se compara.

Lambin (1994) señala que posicionar un producto supone valorarlo por sus características o propiedades más importantes en comparación con los productos de los competidores y respecto a los compradores, para quienes esos elementos de diferenciación son importantes.

De esta manera, el consumidor asigna ciertas posiciones mentales a las marcas por él conocidas, asumiendo, por tanto, el concepto de output perspective de De Chernatony y Dall'Olmo (1997). Este esfuerzo de situación mental de la marca ha de hacerse en base a determinados atributos que juzgan y sitúan a la marca en un lugar (mental) concreto y en relación a otras marcas sobre las que ya asignó determinada posición según tales atributos.

Por otra parte, el perfil de marca preferido por dicho segmento poblacional está representado mediante la denominada "marca ideal". Tal es su importancia que se ha señalado que la intención última de los estudios de posicionamiento es la elaboración de una estrategia adecuada para la empresa, a fin de mejorar su posición actual con relación al ideal (Sanz de la Tajada, 1996: 97). Por lo tanto, la incorporación de dicha marca añade una interesante 
perspectiva estratégica al estudio de posicionamiento de marcas.

Investigaciones previas avalan la importancia del posicionamiento en la estrategia de marketing del sector automotriz (Martínez, Montaner y Pina, 2004; Zamudio, Vega y Bustamante, 2000). Bigné y Vila (2000) realizaron un interesante estudio sobre la estrategia de empresa y posicionamiento de producto en la industria del automóvil, en el que pusieron de manifiesto que los individuos simplifican la interpretación de este mercado definiendo estructuras competitivas compuestas por marcas percibidas como similares.

Hartmann et álli (2004) pusieron de manifiesto que un posicionamiento con base en lo ecológico desde una perspectiva funcional (implementado adecuadamente) podría llevar a una percepción más favorable hacia las marcas de automóviles, en línea con lo señalado por Montero Muradas y Oreja Martínez (2007). Estos últimos autores analizaron el posicionamiento del automóvil todoterreno en España en el 2006 y señalaron como atributos importantes para esta clase de vehículos la seguridad, la habitabilidad y los motores.

Fuera de España, Saavedra Torres et álii (2008), en un estudio de personalidad de las marcas de automóviles, definieron las dimensiones "sinceridad" (dulce, gentil, ingenuo, entusiasta y espiritual), "pacífico" (centrado y elegante), "emocionante" (divertido y atrevido) y "pasional" (apasionado). Omagor y Yuan (2006) estudiaron las similitudes de imagen de la marca Mercedes-Benz en Uganda y Alemania. Loffer (2003) segmentó la demanda de automóviles del mercado alemán usando el análisis Cluster.

Urban, Hoffer y Pratt (2001) estudiaron la incidencia de la percepción de los consumidores del mercado de vehículos usados, poniendo en duda la afectación de la publicidad en dicho mercado.

Por último, Horsky y Nelson (1992), en un estudio realizado sobre un mercado de automóviles norteamericano, mostraron que cuando el consumidor se enfrenta a una elección real, el precio del automóvil es más importante que el resto de factores.

\section{Objetivos}

Los objetivos planteados en este estudio tienen dos vertientes claramente diferenciadas: por un lado, la perspectiva descriptiva, basada en la investigación del posicionamiento de las marcas de automóviles en un grupo poblacional determinado; y, por otro, la perspectiva estratégica, representada mediante la "marca ideal", y que ofrece la visión del "deber ser" de las marcas (desde una concepción perceptual) en dicho grupo poblacional.

Además, se estudió la incidencia de la experiencia en la conducción en la percepción de las marcas, repitiendo el mismo estudio para estudiantes con y sin permiso de conducción. Si bien no puede concluirse experiencia en la conducción por el mero hecho de ser acreedor del permiso administrativo para circular con un vehículo en la vía pública (y tampoco no tenerla aún no disponiendo de tal permiso) parece razonable pensar que aquel que tiene 
dicho permiso está en condiciones de tener experiencia en la conducción y en alguna ocasión ha hecho uso efectivo de tal autorización.

La perspectiva estratégica viene dada, como ya se ha mencionado, por la "marca ideal", que resume las preferencias de la población objeto de estudio en referencia a los atributos estudiados y con relación a las marcas que ocupan tal marco de referencia en dicho espacio perceptual. Al igual que en el caso anterior, se estudia en las circunstancias antes descritas (estudiantes con y sin permiso de conducción).

Así, los objetivos propuestos para este trabajo son los siguientes:

- Análisis del posicionamiento de las marcas de automóviles en 2009.

- Análisis de las diferencias existentes en la percepción (posicionamiento analítico) de las marcas estudiadas en caso de tener o no experiencia en la conducción.

- Análisis desde la perspectiva estratégica (posicionamiento estratégico) de las diferencias existentes en caso de tener o no experiencia en la conducción.

\section{Metodología}

Se seleccionó el universo de estudiantes de una comunidad universitaria específica, la Universidad Europea Miguel de Cervantes, de titularidad privada y con sede en Valladolid (España). Se utilizaron 100 sujetos en el total de la muestra, lo que hace que los resultados obtenidos por la muestra no sean extrapolables al conjunto de estudiantes universitarios, por lo que se presenta como el estudio de un caso concreto, los estudiantes universitarios de la UEMC.

Las marcas elegidas para la evaluación fueron las 14 de mayor volumen de ventas a nivel nacional en 2009. Adicionalmente, se incluyó la "marca ideal"", que resume las preferencias del público objeto de estudio y, por tanto, dota al mapa de una interesante perspectiva estratégica.

Tal y como se ha mencionado, el método de obtención de información fue la encuesta estructurada, en forma de cuestionario autoadministrado. En dicha encuesta se utilizaron esencialmente preguntas dicotómicas, abiertas, cerradas y basadas en escalas. Se determinó el uso de escalas pares ( la dispersión de valores al forzar la decisión en un sentido u otro. Este tipo de escalas ha sido usado en otros análisis de análoga población (Marzal y Calzada, 2003: 57) y también defendidas por otros autores (Cox, 1980: 407; Cafe, Santos, y Macedo, 2001: 73).

En cuanto a la técnica de muestreo, se utilizó el muestreo por cuotas. Se trataba de asegurar la adecuada representatividad del universo, por lo que se reprodujo en dicho muestreo la estructura del universo de estudio en razón de sexo y edad en primer nivel, y tomando en consideración (en segundo lugar) la licenciatura o diplomatura que estudian. El muestreo por cuotas constituye una síntesis de diferentes tipos. En todo caso, ha de considerarse no probabilístico, por lo que en principio no soporta la generalización de lo investigado.

\footnotetext{
1.- Dicho objeto tuvo la consideración de suplementario en el procedimiento estadístico aplicado.
} 
El procedimiento de investigación se ha desarrollado durante el curso lectivo 2008-2010, concretando el trabajo de campo en el mes de abril de 2009. Tal y como se ha mencionado anteriormente, el limitado tamaño de la muestra y la técnica de muestreo utilizada impiden otorgar la consideración de concluyentes a los resultados presentados.

Para la explotación de datos se utilizó el software específico SPSS v. 16. El método de estudio usado para evaluar la percepción en términos de imagen de las marcas analizadas fue el análisis de correspondencias simple con los estadísticos habituales ${ }^{2}$.

\section{Hipótesis de partida}

Considerando los objetivos de conocimiento antes descritos, se proponen las siguientes hipótesis de partida:

- La percepción del mercado de automóviles se estructura en dos grupos competitivos en función principalmente del precio de las marcas, con independencia del factor experiencia en la conducción.

- Las preferencias del público objeto de estudio, en relación a las marcas contempladas, se ubican en torno a las marcas de gama media, con independencia del factor experiencia en la conducción.

\section{Resultados}

Se determinó usar las marcas Audi, BMW, Citroën, Fiat, Ford, Hyundai, Mercedes Benz, Opel, Peugeot, Renault, Seat, Toyota y Volkswagen por ser las que acumularon los mayores volúmenes de unidades vendidas en España (Dirección Nacional de Tráfico, 2010). En efecto, el hecho de contemplar únicamente estas marcas, supone considerar el $88.22 \%$ de dichas ventas en el conjunto de España, lo que estructura de forma importante la oferta de automóviles.

Los atributos que se seleccionaron para evaluar las marcas se determinaron a partir del estudio de orden cualitativo mencionado anteriormente. Fueron finalmente seleccionados:

- Fiabilidad. Se averigua si los estudiantes universitarios consideran que las marcas en cuestión fabrican coches resistentes y fiables.

- Estética. Se investiga si las marcas en cuestión fabrican automóviles estéticamente adecuados para los estudiantes universitarios. Se trata de averiguar la dimensión estética, lo bonito o feo de un automóvil y su importancia en el proceso de decisión de compra.

- Seguridad. Se constata aquí en qué grado los estudiantes creen que las marcas testadas fabrican automóviles seguros. Se incluye aquí la seguridad como atributo genérico, es decir, incluye tanto la seguridad pasiva como la seguridad activa.

- Equipamiento y calidad de los acabados. Se investiga en qué grado los estudiantes

2.- Medida de distancia Chi-cuadrado, método de normalización simétrico. Se decidió el uso de dos dimensiones y se elaboró un gráfico de dispersión biespacial en todos los casos analizados. 
universitarios juzgan la calidad de los materiales en que está fabricado el automóvil. El equipamiento hace referencia tanto a la calidad del equipamiento como a su cantidad.

- Consumo. Se averigua en qué grado juzgan los estudiantes que consumen combustibles los automóviles de las diferentes marcas en cuestión.

- Potencia. Se investiga en qué grado juzgan los estudiantes universitarios la potencia que las marcas incluyen en sus automóviles.

- Contaminación. Se investiga en qué grado son percibidas como contaminantes las marcas que incluyen este estudio.

- Prestigio del país de origen. Se estudia el grado de prestigio del país de origen adjudicado a las marcas que incluyen este estudio, así como el grado de conocimiento de los países de origen de las marcas sometidas a estudio.

- Precio. Dada la (a priori) escasa capacidad económica de los estudiantes universitarios, se investiga la percepción de esfuerzo económico que para los estudiantes universitarios supone la adquisición de cierta marca de automóvil.

- Prestigio social de la marca. Se averigua la percepción de consideración social de las marcas participantes en el estudio. Este atributo se enlaza con la idea de la marca del automóvil y del propio automóvil como muestra de status social.

- Período de garantía. Se investiga la percepción de los estudiantes universitarios acerca del período de garantía ofrecido por las marcas participantes y su grado de conocimiento.

- Prescriptores de opinión. Se averigua el grado de conocimiento de los estudiantes acerca de la opinión que las revistas especializadas guardan acerca de las marcas objeto de estudio.

\subsection{Todos los casos}

Considerado el colectivo objeto de estudio, podemos concluir que los estudiantes universitarios de la UEMC ordenan y clasifican las marcas en función del precio atribuido a ellas.

De hecho, en el mapa, las variables que explican la posición atribuida a las marcas estudiadas son los atributos "baratos" y "para la clase alta" los que efectivamente ordenan y clasifican las marcas en tres grandes grupos competitivos: a) las marcas de "gama media", explicado por su cercanía al atributo "barata" y su lejanía al atributo "para la clase alta"; b) las marcas en torno a un "segmento Premium", en sentido contrario, explicado por su lejanía al atributo "barata" y su cercanía al atributo "para la clase alta"; c) una marca con un "perfil único", original, de transición entre un grupo y el otro. Los ejes para este mapa estarían conceptualizados en el binomio "barato-para la clase alta" en la dimensión 1 (que es la dominante) y la dimensión 2, en "lo más o menos reputado"3.

Así, tal y como puede apreciarse en la figura $1^{4}$, el segmento "generalista" estaría formado

3.- Esta dimensión es mucho más débil que la primera.

4.- La leyenda para el mapa realizado de los atributos analizados es "barato" (1), "consume" (2), "contamina" (3), "buenos acabados y equipamiento" (4), "bonitos" (5), "fiables" (6), "con amplio período de garantía" (7), "para la clase alta" (8), "potente" (9), "de origen prestigioso" (10), "recomendada por las revistas especializadas" (11) y "seguros" (12). Las marcas están representadas por las siguientes iniciales: Audi (AU), BMW (BMW), 
por las marcas Citroën, Fiat, Ford, Hyundai, Opel, Peugeot, Renault, Seat y Toyota. Las marcas pertenecientes al segmento Premium serían Audi, BMW y Mercedes Benz en el mapa; y la marca de transición entre los dos grupos sería Volkswagen.

En este mismo contexto, la "marca ideal" (y, por tanto, las preferencias declaradas del colectivo objeto de estudio) se encuentra situada en el entorno de las marcas generalistas.

Figura 1: Diagrama de dispersión biespacial. Todas las marcas.

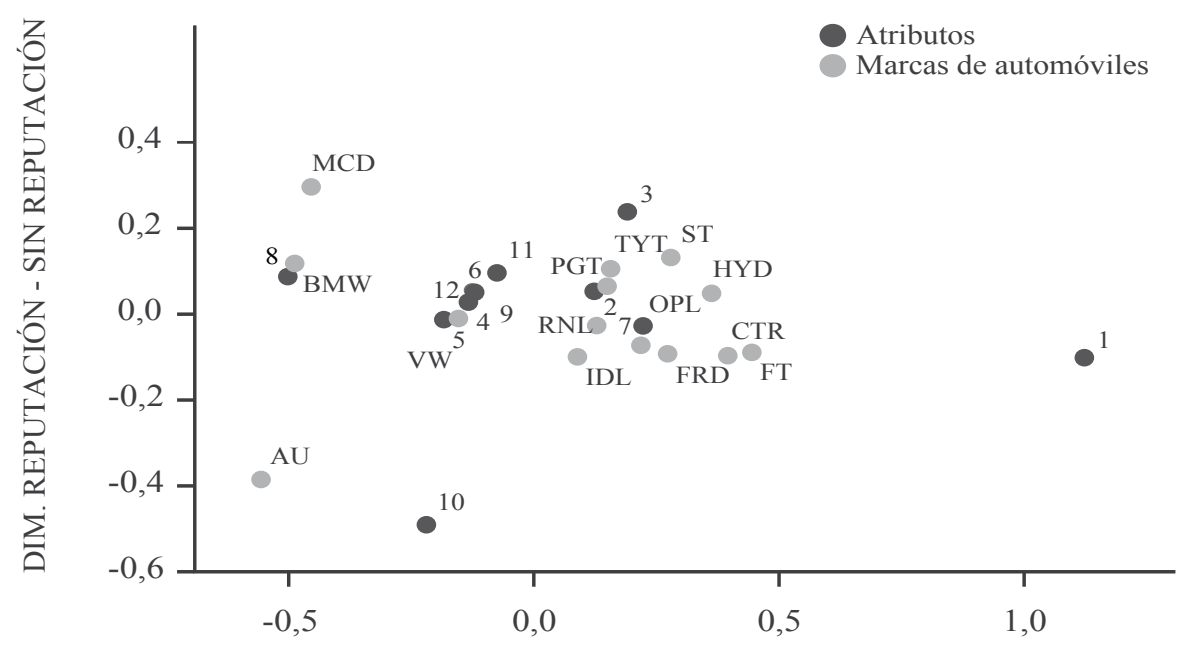

DIMENSIÓN EXCLUSIVO - POPULAR

Fuente: Elaboración propia con SPSS.

El dominio de los atributos "barato" y "para la clase alta" (los cuales dominan los mapas y determinan la posición de los restantes atributos) obligó el desdoblamiento del análisis en dos más: por un lado, se realizó un análisis con las marcas percibidas en un segmento Premium y Volkswagen; y, por otro lado, un análisis con las marcas generalistas y Toyota.

Con referencia a las marcas ubicadas en el segmento Premium, se debe acotar que el análisis realizado ${ }^{5}$ permite concluir que estas son consideradas marcadamente caras, adscritas a grupos sociales altos y excelentes, según todos los atributos testados. Dicha excelencia (y su lejanía con la marca ideal) permite sugerir que son marcas que actúan como referentes sociales. Es posible que (dada la a priori limitada condición económica de los estudiantes universitarios) estas marcas sean consideradas como referentes de éxito social y económico, pues el mero hecho de ser propietario de un automóvil de estas marcas supone la adscripción a un grupo social alto (por su marcada adscripción al atributo "para la clase alta") y a una

Citroën (CTR), Fiat (FT), Ford (FRD), Hyundai (HYD), Mercedes Benz (MCD), Opel (OPL), Peugeot (PGT), Renault (RNL), Seat (ST), Toyota (TYT) y Volkswagen (VW).

5.- No se incluye el gráfico de dispersión biespacial al no aportar mayor claridad en la descripción de las posiciones declaradas de las marcas. 
posición económica desahogada (por su marcada lejanía al atributo “barato”).

En cualquier caso, se puede concluir que el colectivo objeto de estudio considera que las marcas Audi, BMW y Mercedes son muy similares entre sí, considerando todos los atributos testados.

El análisis realizado sobre las marcas generalistas, cuyo resultado en forma de mapa de posicionamiento puede verse en la figura $2^{6}$, permiten señalar interesantes diferencias perceptuales respecto a las marcas estudiadas.

Figura 2: Diagrama de dispersión biespacial. Marcas de gama media.

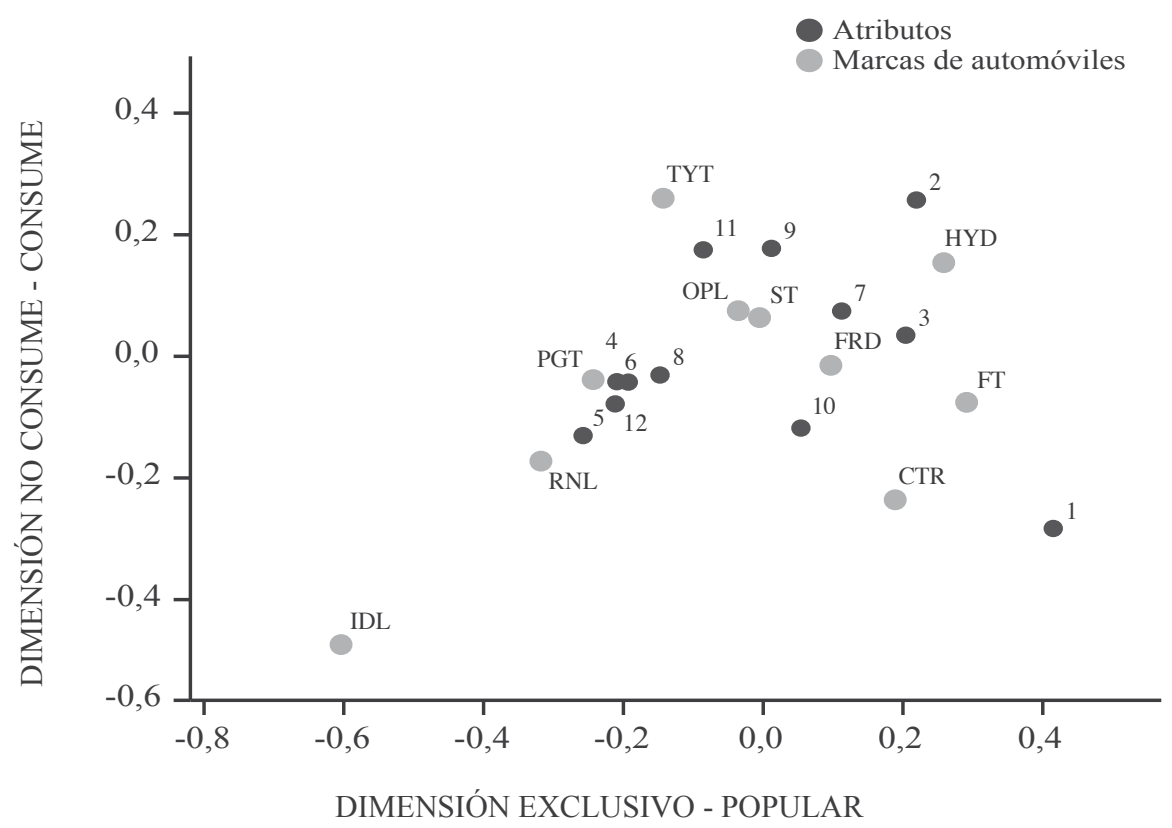

Fuente: Elaboración propia con SPSS.

Así, en el mapa puede observarse que Citroën es percibida como una marca "barata" de escaso consumo. Seat muestra un perfil muy similar a Opel y es percibida esencialmente como una marca que no es barata, pero que es "potente y consume", con amplio período de garantía y que goza de los favores de la prensa especializada. Además, se encuentra alejada del ideal, lo que sugiere no ser la opción de compra preferida por los estudiantes de la UEMC.

6.- Al igual que en el gráfico anterior, para el mapa la leyenda de los atributos analizados es "barato" (1), "consume" (2), "contamina" (3), "buenos acabados y equipamiento" (4), "bonitos" (5), "fiables" (6), "con amplio período de garantía" (7), "para la clase alta" (8), "potente" (9), "de origen prestigioso" (10), "recomendada por las revistas especializadas" (11) y "seguros" (12). Igualmente, las marcas están representadas por las siguientes iniciales: Citroën (CTR), Fiat (FT), Ford (FRD), Hyundai (HYD), Opel (OPL), Peugeot (PGT), Renault (RNL), Seat (ST) y Toyota (TYT). 
Fiat forma un grupo competitivo con Citroën y Hyundai. De ellas, las más parecidas entre sí son Fiat y Citroën, que no muestran vinculación con el consumo elevado.

Hyundai muestra cierta relación con un consumo elevado, y también (aunque mucho más difusa) con el atributo "potente" y, en cierta medida, con el elevado período de garantía. Toyota es percibida como una marca "potente" de alto consumo y no barata, pero sin vinculación al atributo para la clase alta.

La figura 2 también muestra dos marcas percibidas como muy similares entre sí en función de los atributos testados: Peugeot y Renault, las cuales son consideradas como marcas "bonitas", con buenos acabados, buen nivel de equipamiento y fiables. Además, muestran vinculación con el atributo para la clase alta, que hace posible sugerir cierta labor de "marca puente" entre la gama media y Volkswagen. No obstante, el trabajo de campo se hizo en Valladolid, ciudad en la que Renault tiene una fábrica, por lo que la percepción de esta marca en dicho entorno ha de ser cuestionada, ya que al ser una marca que tiene gran importancia en la economía local es posible que haya sido sobrevalorada.

\subsection{Diferencias de percepción en función de la tenencia o no de permiso de conducción}

Tal y como se ha mencionado anteriormente, se ha considerado el estudio diferenciado de la muestra según tuviera o no de permiso de conducción. Se trata en este punto de determinar si la simple tenencia, y consecuentemente la experiencia en la conducción, altera de alguna forma la percepción de las marcas consideradas en el presente estudio. Los resultados se muestran a continuación.

\subsubsection{Posicionamiento de marcas en usuarios sin permiso de conducción}

Los estudiantes sin permiso de conducción muestran, consideradas todas las marcas analizadas, la misma situación estructural vista anteriormente ${ }^{7}$. Del mismo modo, las variables que explican la posición atribuida a las marcas estudiadas son los atributos "barato" y "para la clase alta", que efectivamente ordenan y clasifican las marcas en tres grandes grupos competitivos: $a$ ) las marcas de gama media ${ }^{8}$, explicado por su cercanía al atributo "barata" y su lejanía al atributo "para la clase alta"; $b$ ) las marcas en torno a un segmento Premium 9 , en sentido contrario, explicado por su lejanía al atributo "barata" y su cercanía al atributo "para la clase alta"; y c) marca de transición (Volkswagen) entre un grupo y el otro.

La marca ideal (y por tanto las preferencias declaradas del colectivo objeto de estudio) también se encuentra situada en el entorno de las marcas de gama media. Al igual que en el caso anterior, el amplio dominio de los atributos para la clase alta y baratos hizo obligado el desdoblamiento del análisis en los dos también antes mencionados.

De nuevo, el análisis del posicionamiento de marcas de gama alta puso de relevancia su consideración de marcas "caras-excelentes" según todos los atributos testados, y de un perfil muy similar, por lo que también en este caso cabe sugerir su posición como objetos de referencia.

Sin embargo, se apreciaron diferencias interesantes en el segmento generalista, tal y como

\footnotetext{
7.- No se muestra el gráfico de dispersión biespacial al no añadir claridad en la exposición.

8.- Este grupo estaría formado por las marcas Citroën, Fiat, Ford, Hyundai, Opel, Peugeot, Renault, Seat y Toyota.

9.- Estaría formado por las marcas Audi, BMW y Mercedes Benz.
} 
puede apreciarse en la figura $3^{10}$.

Figura 3: Diagrama de dispersión biespacial. Marcas de gama media.

Usuarios sin permiso de conducción.

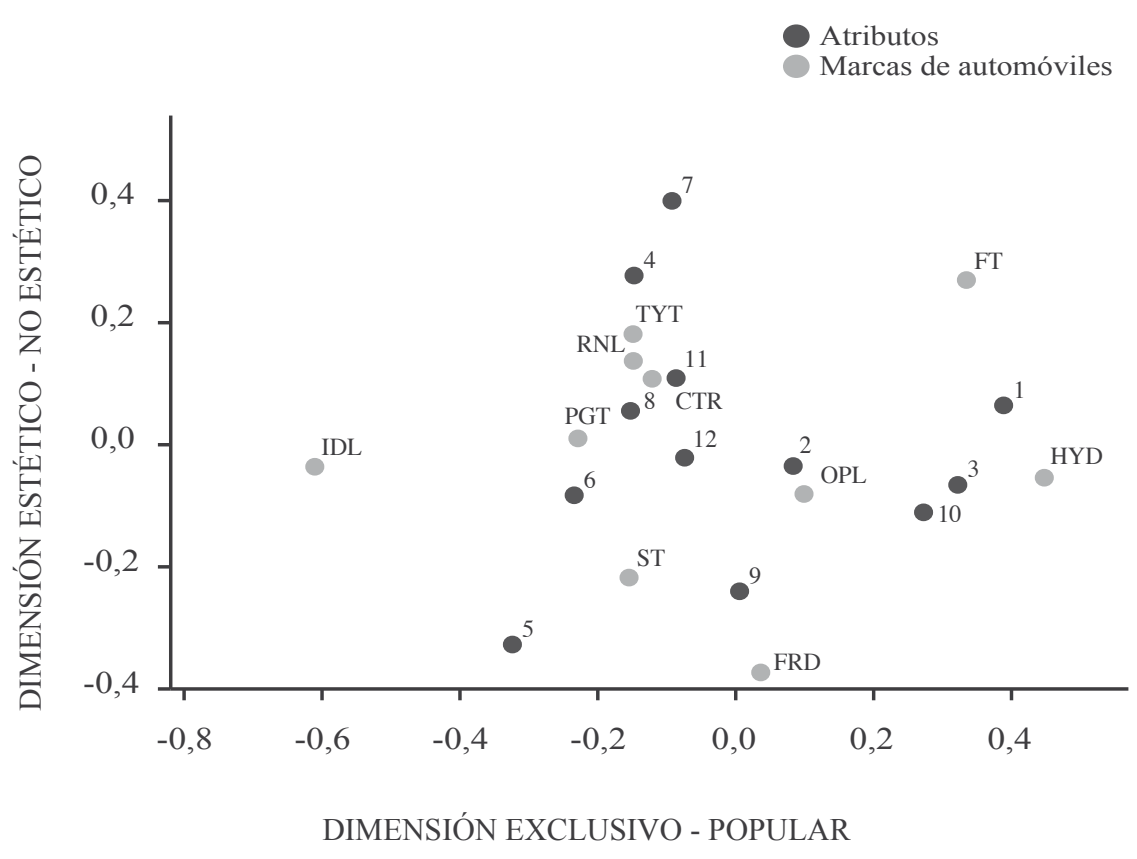

Fuente: Elaboración propia con SPSS.

En el mapa se observa un grupo competitivo formado por Toyota, Renault, Citroën y Peugeot. Toyota está en dicho contexto especialmente asociado a los atributos "buenos acabados y equipamiento" y "amplio período de garantía”. Renault y Citroën muestran un perfil muy similar a Toyota, aunque estas presentan cierta asociación con las variables "para la clase alta" y "recomendada por las revistas especializadas". Peugeot es una marca peculiar dentro de ese grupo competitivo, con especial vinculación a la "fiabilidad", además del atributo "para la clase alta".

Hyundai y Fiat comparten la posición de marcas "baratas" y "que contaminan", sin asociación al resto de atributos. Opel y Ford, que comparten cuadrante, se caracterizarían por el "alto consumo" y "alta potencia". Opel además muestra vinculación con el prestigio del país de origen, que podría explicarse en la tradicional vinculación del sector a Alemania.

10.- Al igual que en los gráficos anteriores, para el mapa la leyenda de los atributos analizados es "barato" (1), "consume" (2), "contamina" (3), "buenos acabados y equipamiento" (4), "bonitos" (5), "fiables" (6), "con amplio período de garantía" (7), "para la clase alta" (8), "potente" (9), "de origen prestigioso" (10), "recomendada por las revistas especializadas" (11) y "seguros" (12). Igualmente, las marcas están representadas por las siguientes iniciales: Citroën (CTR), Fiat (FT), Ford (FRD), Hyundai (HYD), Opel (OPL), Peugeot (PGT), Renault (RNL), Seat (ST) y Toyota (TYT). 
Por último, Seat se muestra como una marca con cierta indefinición perceptual, con débiles asociaciones a lo "bonito", "fiable" y "potente". Además, cabe sugerir que esta marca no constituye una opción preferente de consumo, pues en el mapa se observa su alejamiento de la marca ideal.

\subsubsection{Posicionamiento de marcas en usuarios con permiso de conducción}

Los estudiantes con permiso de conducción muestran, considerando todas las marcas, la misma situación estructural repetida anteriormente, es decir, un grupo de marcas Premium (Audi, BMW y Mercedes Benz), otro de gama media (el resto de marcas salvo Volkswagen) y una marca puente entre estas y aquellas (Volkswagen). Igualmente, en este caso, la variable crítica es el precio, que estructura el mercado y define dichos grupos competitivos, por lo que nuevamente se hizo obligado el desdoblamiento del análisis.

Al igual que en los casos anteriores, el análisis del posicionamiento de marcas de gama alta puso de relevancia su consideración de marcas caras y "excelentes", según todos los atributos testados, y de un perfil muy similar, por lo que también cabe sugerir su posición como objetos de referencia. Otra vez, pudieron apreciarse interesantes diferencias en el segmento de gama media ${ }^{11}$.

Figura 3: Diagrama de dispersión biespacial.

Marcas de gama media. Usuarios con permiso de conducción.

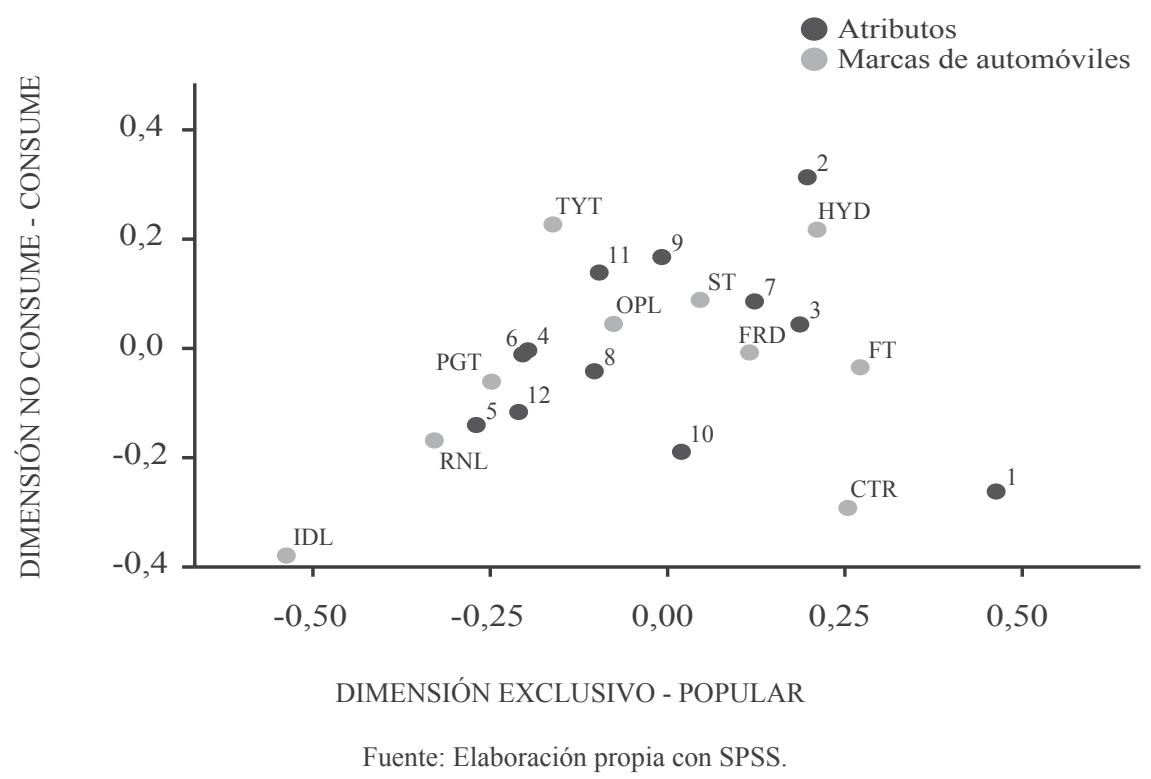

11.- Al igual que en los gráficos anteriores, para ambos mapas la leyenda de los atributos analizados es "barato" (1), "consume" (2), "contamina" (3), "buenos acabados y equipamiento" (4), "bonitos" (5), "fiables" (6), "con amplio período de garantía" (7), "para la clase alta" (8), "potente" (9), "de origen prestigioso" (10), "recomendada por las revistas especializadas" (11) y "seguros" (12). Igualmente, las marcas están representadas por las siguientes iniciales: Citroën (CTR), Fiat (FT), Ford (FRD), Hyundai (HYD), Opel (OPL), Peugeot (PGT), Renault (RNL), Seat (ST) y Toyota (TYT). 
En el mapa (figura 4) también se aprecia que las marcas Fiat, Hyundai y Citroën constituyen el grupo de marcas percibidas como las más baratas en ese contexto, que consumen y contaminan, pero no muestran vinculación con "potencia", por lo que cabe sugerir cierta ineficiencia tecnológica en dichas marcas.

Las marcas Renault, Peugeot, Toyota y Opel forman una estructura, vinculada a los atributos "para la clase alta", "potencia", "buenos acabados y el amplio equipamiento", "fiabilidad" y "estética". Formarían, por tanto, el grupo de marcas menos orientadas a precio y más orientadas a valor. En dicho grupo se encuentra la "marca ideal".

Por ello, cabe sugerir que este colectivo percibe a Toyota como una marca que realiza funciones de "marca puente" entre el segmento medio y Volkswagen. Cabe también señalar que las marcas Peugeot, Opel y Renault se adscriben a este mismo grupo, si bien en el caso de Renault hay que considerar su posible sobrevaloración por las razones antes expuestas.

Seat y Ford forman otro micro grupo competitivo, asociado en mayor medida al "amplio período de garantía" y, en menor medida, a la "alta contaminación". Forman un grupo de precio medio en dicho contexto.

\section{Conclusiones}

Los análisis efectuados determinan que el mercado se estructura para el colectivo objeto de estudio en función del precio. Además, no se pudo determinar diferencia alguna de percepción al considerar la tenencia o ausencia de permiso de conducción. Por ello, en todos los casos debe concluirse que el precio es el factor fundamental en la ordenación perceptual de las marcas en estructuras competitivas.

Así, la lejanía de dicha variable y la cercanía de la asociada al prestigio social de la marca (esto es, el atributo "para la clase alta") determina la existencia de un grupo competitivo Premium, formado por las marcas Audi, BMW y Mercedes Benz. Dichas marcas son percibidas como muy similares entre sí y excelentes según todos los atributos testados. Además, están alejadas de la variable "barato" y muy próximas al atributo "para la clase alta". Finalmente, se encuentran muy alejadas de la "marca ideal", lo que hace sugerir que estas marcas actúan como objetos de referencia y no como una opción real de consumo para el colectivo estudiado.

En la posición antagónica se encontraría otra estructura competitiva formada por las marcas Citroën, Fiat, Ford, Hyundai, Opel, Peugeot, Renault, Seat y Toyota. Esta estructura estaría ligada al precio bajo y también escasa asociación al prestigio social, por lo que formaría el segmento de gama media. Se pudieron constatar diferencias tanto de percepción de las marcas implicadas en dicho segmento, como su ordenación en microestructuras competitivas con perfiles especialmente similares.

Finalmente, se pudo determinar la existencia de una marca con un perfil perceptual único y original. Se trata de la marca Volkswagen, que se encontraría entre las dos estructuras anteriores, asumiendo una posición de transición entre ambas. 
Habida cuenta de lo antes señalado, no se pudo verificar la hipótesis primera, ya que se pudo determinar la existencia de tres grupos competitivos (y no dos como en principio se sospechaba) si bien la ordenación en términos de percepción de dichas marcas obedece, en todos los casos, a la variable precio.

La "marca ideal", que proporciona visión estratégica en el mapa, muestra que para todos los colectivos estudiados, el segmento preferente de consumo (para el colectivo objeto de estudio) es el de las marcas de gama media, compatible con la limitación presupuestaria que, en principio, debería atribuirse a este colectivo, si bien se observa que no se pretende únicamente una marca barata, sino una "razonablemente barata" pero con "equipamiento y buenos acabados", "estética", "fiabilidad", entre otros.

Así, la segunda hipótesis que se planteaba pudo verificarse, ya que las preferencias del colectivo objeto de estudio en todos los casos se sitúan en torno a las marcas de gama media.

Por todo ello, cabe suponer que una estrategia basada exclusivamente en políticas agresivas en torno al precio no colmaría los deseos de este grupo poblacional, por lo que habría que considerar la posibilidad de incluir elementos adicionales que incrementen el valor percibido de la marca y mejorar así su propuesta de valor. No obstante, lo anterior no debe en ningún caso descuidarse del factor "precio", ya que este colectivo es muy sensible a dicha variable, por lo que el punto de inicio de una hipotética decisión de compra de estos bien podría atender a este factor.

Entre las limitaciones más importantes de este trabajo hay que mencionar el carácter descriptivo y estratégico del estudio, que no ofrece explicación alguna sobre el hecho estudiado, es decir, se conoce la percepción del colectivo objeto de estudio sobre las marcas de automóviles y el posicionamiento deseado, pero no se ofrecen explicaciones sobre el hecho, ni sobre los factores que han motivado tal percepción. Además, el objeto de estudio se basa en un caso específico y referente a un sector de la industria concreta, por lo que es imposible generalizar los resultados obtenidos.

Como futuras líneas de trabajo, cabría apuntar la posibilidad de realizar análisis explicativos sobre este sector, relacionando el posicionamiento de las marcas con diversas variables tanto de marketing como de otra naturaleza, a fin de intentar establecer relaciones causales que mejoren la eficiencia del desempeño empresarial en este sector. Podría desplazarse tal estudio a otros sectores industriales relacionados o bien contemplar distintos escenarios culturales que podrían ser fuente de interesantes reflexiones al respecto.

\section{Fuentes consultadas}

Bigné J. E. y Vila, N. (2000). "Estrategia de empresa y posicionamiento de producto en la industria del automóvil. Un estudio comparado”. Economía Industrial, 332, 29-38.

Cafe, L., Santos, C. y Macedo, F. (2001). "Proposta de um método para escolha de software de automação de bibliotecas”. Ci. Inf., mayo-agosto, 30(2), 70-79. 
Cox, E. P. (1980). “The Optimal Number of Responses Alternatives for a Scale: A Review”, Journal of Marketing Research, 17, 407-422.

De Chernatony, L. y Dall'Olmo (1997). “The chasm between managers and consumers views of brands: the experts perspectives". Journal of Marketing Management, 13, 89-104.

Díaz de Alda, J. (2009, Noviembre 11) "La automoción Española, en manos de gobiernos extranjeros y ayudas públicas". La Verdad.es. Extraída el 13/XI/2009 desde http://www.laverdad.es/ albacete/20091011/economia/automocion-espanola-manos-gobiernos-20091011.html.

Dirección General de Tráfico (2010). Anuario Estadístico General 2009. Madrid: DGT.

Hartmann, P. et álii. (2004). "Superando los límites medioambientales de la empresa: un estudio experimental del efecto del posicionamiento ecológico en la actitud hacia la marca". Cuadernos de gestión, 4 (1), 3-85.

Horsky, D. y Nelson, P. (1992). "New brand positioning and pricing in an oligopolistic market". Marketing Science, 11 (2), 133-153.

Joachimstaler, E. y Aaker, D. (2005). "Crear marcas sin utilizar medios de comunicación masivos". En Harvard Bussiness Review (ed.). Gestión de Marcas. Bilbao: Deusto.

Kotler, P. y Keller, K. L. (2007). Dirección de Marketing. Madrid: Pearson Education.

Lambin, J. J. (1994). Marketing estratégico. Madrid: McGraw Hill.

Loffer (2003). "Market-segments of automotive brands: Letting multivariate analyses reveal additional insights", pp. 492-503. 25th Annual Conference of the German-Classification-Society, Studies in classification, data analysis and knowledge organization. Munich: Universidad de Munich.

Martínez Salinas, E.; Montaner Gutiérrez, T. y Pina Pérez, J. (2004). "Propuesta de una metodología. Medición de la imagen de marca. Un estudio exploratorio”. ESIC-Market, 1, 200-216.

Marzal García-Quismondo, M. A. y Calzada Prado, F. J. (2003). "Un análisis de necesidades y hábitos informativos de estudiantes universitarios en Internet". Binaria: Revista de comunicación, cultura y tecnología, 3,57 y ss.

Montero Muradas, I. y Oreja Martínez, J. R. (2006). "El posicionamiento del automóvil todoterreno en España. Un análisis conjunto multiatributo", pp. 134-145. En Febles, J. y Oreja, J. R. (eds.). Modelos de Rasch en Administración de Empresas. Santa Cruz de Tenerife: FYDE-CajacanariasColección e-books.

Montero Muradas, I. y Oreja Martínez, J. R. (2007). "Benchmarking competitivo del consumo entre automóviles en el mercado español". Instituto Universitario de la Empresa, Serie Estudios, 59, 1-15.

Oficina de Justificación de la Difusión (2009). "Listado de revistas". Extraída el 13/XI/2009, desde http://www.ojd.es

Omagor, S. y Yuan, G. H. (2006). "A comparison on marketing strategies for Mercedes between Germany and Uganda", pp. 807-810. Proceedings of the 3rd International Conference on Innovation \& Management.

Saavedra Torres, J. L. et álii. (2008). "Medición de la personalidad de marca en el mercado automotriz". Visión Gerencial, 1, 183-196. 
Sanz de la Tajada, L. A. (1996). Auditoría de la imagen de la empresa. Métodos y técnicas de estudio de la imagen. Madrid: Síntesis.

Trout, J. y Ries, A. (1972). “The positioning era comet”. Advertising Age, 24, 35-38.

Trout, J. y Rivkin, S. (2001). Diferenciarse o morir. Cómo sobrevivir en un entorno competitivo de alto riesgo. Madrid: McGraw Hill.

Urban, D. J.; Hoffer G. E. y Pratt M. D. (2001). "Perceptions of advertising in the market for used vehicles“. Journal of Advertising Research, 41(1), 66-77.

Zamudio Wehrhahn, X.; Vega Gómez, J. y Bustamante Ubilla, M. (2000). Estudio de mercado: monitoreo de marcas en el consumidor de automóviles de pasajeros [Tesis]. Chile: Universidad de Talca. 